\title{
DETECÇÃO DA ENCEFALOPATIA HEPÁTICA SUBCLÍNICA POR ESPECTROSCOPIA CEREBRAL
}

\author{
Detection of subclinical hepatic encephalopathy by magnetic resonance \\ spectroscopy
}

\author{
Gustavo Justo SCHULZ, Julio Cézar Uili COELHO, Jorge Eduardo Fouto MATIAS, \\ Antônio Carlos Ligocki CAMPOS, Danielle Duck SCHULZ, Guilherme Augusto BERTOLDI
}

ABCDDV/532

Schulz GJ, Coelho JCU, Matias JEF, Campos ACL, Schulz DD, Bertoldi GA. Detecção da encefalopatia hepática subclínica por espectroscopia cerebral. ABCD Arq Bras Cir Dig 2007; 20(2):81-6.

RESUMO - Racional - A encefalopatia hepática é anormalidade neuropsiquiátrica comum em cirróticos e está associada com alterações típicas de determinados metabólitos cerebrais, como o decréscimo de mio-inositol e colina e o aumento de glutamina-glutamato, observadas na espectroscopia cerebral por ressonância magnética. Objetivo - Determinar os níveis dos metabólitos cerebrais em pacientes cirróticos para diagnóstico da encefalopatia hepática em estágios iniciais. Métodos - Foram estudados 25 pacientes com cirrose hepática, do Serviço de Transplante Hepático da Universidade Federal do Paraná, através de avaliação clínica (exame neurológico e testes neuropsicométricos) e espectroscopia por ressonância magnética cerebral. A área espectral estudada por ressonância magnética envolveu a região occipital (substância branca e cinzenta). Trinta voluntários sadios formaram o grupo controle. Resultados - A encefalopatia hepática subclínica foi diagnosticada em 12 pacientes (48\%). Reduções significativas nos índices de $\mathrm{MI} / \mathrm{Cr}$ foram observadas nos pacientes com encefalopatia quando comparados aos controles $(0,49 \pm 0,10$ vs. $0,83 \pm 0,13$; $P<0,0001)$. Em adição, os pacientes com encefalopatia mostraram redução no índice $\mathrm{Cho} / \mathrm{Cr}(0,54 \pm 0,15$ vs. $0,77 \pm 0,09 ; P=0,0001)$ e aumento no índice de $\mathrm{Glx} / \mathrm{Cr}(1,12 \pm 0,26$ vs. $0,89 \pm 0,19 ; P=0,0146)$ quando comparados ao grupo controle. Os critérios quantitativos de Ross para diagnóstico espectroscópico da encefalopatia hepática (MI/Cr e Cho/Cr $<$ média + 2 desvios-padrão do grupo controle) demonstraram sensibilidade de $61,54 \%$, especificidade de $91,67 \%$ e precisão de $76 \%$. O índice Cho/Cr foi o melhor parâmetro isolado. Conclusão - As alterações metabólicas cerebrais que ocorrem precocemente no curso da encefalopatia hepática podem ser demonstradas pela espectroscopia, permitindo diagnóstico preciso antes mesmo do aparecimento de sinais e sintomas clínicos.

DESCRITORES - Encefalopatia hepática. Encefalopatias. Espectroscopia de ressonância magnética.

\section{INTRODUÇÃO}

Os distúrbios neuropsiquiátricos associados à doença hepática crônica foram descritos, pela primeira vez, há mais de 2000 anos por Hipócrates. Apesar disto, os mecanismos precisamente envolvidos com o seu desenvolvimento permanecem incertos. A encefalopatia hepática pode ocorrer tanto na insuficiência hepática aguda quanto na crônica, mais comumente associada à disfunção hepática e derivações portossistêmicas.

A prevalência da encefalopatia hepática em cirróticos varia de $30 \%$ a $84 \%$ e pode ocorrer em mais de $80 \%$ dos pacientes submetidos à derivação portocava cirúrgica ${ }^{22}$. A encefalopatia é comumente subestimada em virtude da preservação das habilidades verbais dos pacientes em estádios iniciais da doença, observando-se apenas lentificação no tempo de reação nas atividades cotidianas deixandoos inseguros, por exemplo, para dirigir automóvel. Esta forma de encefalopatia está presente na maioria dos cir-

Trabalho realizado na Disciplina de Cirurgia do Aparelho Digestivo do Setor de Ciências da Saúde da Universidade Federal do Paraná, Curitiba, PR, Brasil

Endereço para correspondência: Gustavo Justo Schulz, e-mail: gjschulz@hotmail.com róticos. Nas situações clinicamente aparentes, a síndrome apresenta manifestações mais severas, com alterações de comportamento, personalidade e distúrbios no nível de consciência.

A literatura mostra evidências cada vez mais consistentes sobre a importância do diagnóstico e terapêutica precoce da encefalopatia hepática, preferencialmente antes do aparecimento de sinais clínicos evidentes, visando a preservação das funções cerebrais, pois alguns autores, contrariando o caráter metabólico reversível da síndrome, acreditam tratar-se de desordem neurodegenerativa lentamente progressiva ${ }^{12}$.

Na prática clínica rotineira, a tomografia computadorizada tem sido usada para excluir outros diagnósticos, como hematomas subdurais, que também podem causar alteração do nível de consciência em pacientes cirróticos. Até recentemente, a ressonância magnética era considerada como exame de segunda linha na investigação da encefalopatia em muitos países, assim como a espectroscopia cerebral por ressonância magnética estava confinada a instituições de pesquisa, revelando significativos avanços no entendimento dos mecanismos fisiopatológicos envolvidos. Melhorias tecnológicas no campo da ressonância 
têm permitido aumento da resolução e sensibilidade do método, fornecendo diagnósticos cada vez mais precoces da doença.

Os objetivos deste estudo foram mostrar as alterações espectroscópicas nos metabólitos cerebrais em estágios precoces da encefalopatia hepática em cirróticos e também os índices de qualidade da espectroscopia no diagnóstico da doença.

\section{MÉTODOS}

No período compreendido entre outubro de 2002 e novembro de 2003 foram estudados, por meio de avaliação clínica e espectroscopia cerebral por ressonância magnética, pacientes com doença hepática crônica com idade acima de 15 anos, candidatos à transplante no Serviço de Transplante Hepático do Hospital de Clínicas da Universidade Federal do Paraná, em Curitiba, PR.

Foram estudados 25 pacientes, sendo 19 (76\%) do sexo masculino e $6(24 \%)$ do feminino. A principal causa de disfunção hepática foi hepatite C em 11 casos (44\%), seguida pelo álcool em 6 (24\%), hepatite B em 5 (20\%), hepatite auto-imune em $1(4 \%)$, colangite esclerosante primária em $1(4 \%)$ e esteatohepatite não-alcoólica em 1 (4\%). Dois pacientes $(8 \%)$ apresentavam hepatocarcinoma associado. A maioria (72\%) era Child-Pugh B. A história de encefalopatia hepática estava presente em sete pacientes (28\%).

Trinta voluntários sadios formaram o grupo controle. Dezesseis (53,33\%) eram do sexo masculino e 14 (46,67\%) do feminino.

A avaliação clínica, tanto de pacientes como dos controles, foi realizada próxima à avaliação espectroscópica (diferença entre as duas avaliações não superior a 48 horas).

A encefalopatia hepática foi diagnosticada observandose os dados do exame clínico, exame neurológico e dos testes neuropsicométricos. Para graduação clínica utilizou-se os critérios de Parsons-Smith ${ }^{19}$ e para a disfunção hepática a classificação de Child-Pugh. A avaliação das funções cognitivas foi realizada por meio de testes neuropsicométricos, que incluíram: mini-exame do estado mental ${ }^{3,5}$, teste de trilhas A e $\mathrm{B}^{15}$, subteste de dígitos da bateria WAIS-R ${ }^{31}$, teste de fluência verbal (FAR) e categorias ${ }^{15}$ e teste do desenho do relógio ${ }^{15}$.

A espectroscopia cerebral foi realizada com um aparelho de ressonância magnética com 1,5T (Magnetom Vision; Siemens, Erlanger, Germany), utilizando-se uma bobina polarizada circular posicionada junto à cabeça dos examinados. A exata reprodução da localização espectral foi conseguida mediante a aquisição prévia de três cortes (sagital, transversal e coronal) na ressonância magnética em T2 (tempo de relaxamento transversal dos núcleos) utilizando-se a técnica de "fast spin echo" para posicionamento tridimensional do volume de interesse ("voxel") na parte medial do lobo occipital, que envolve substância branca e cinzenta, com 2 X 3 X $2 \mathrm{~cm}^{3,18}$ (Figura 1).

Para detecção e quantificação do MI e Glx foram

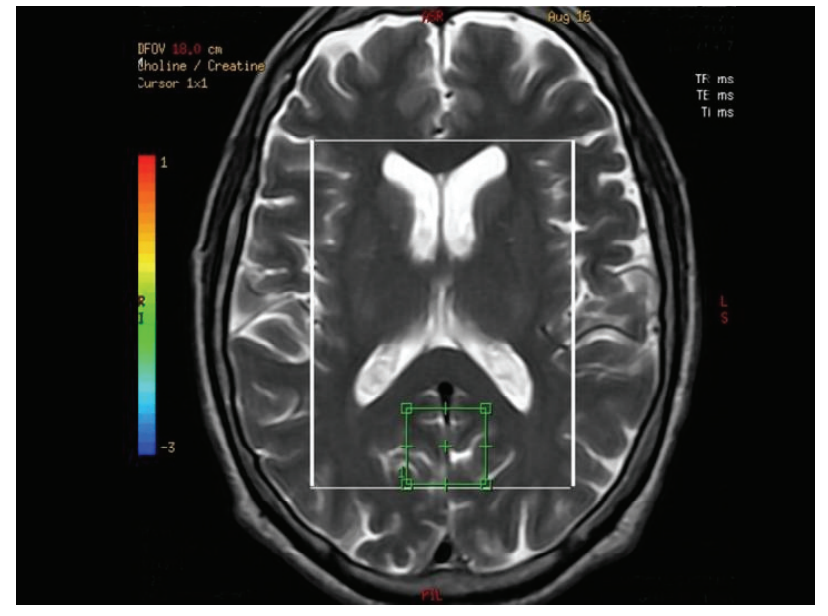

* NOTA: volume de interesse $=2 \times 3 \times 2 \mathrm{~cm} 3$

FIGURA 1 - Localização do volume de interesse ("voxel") na porção medial do lobo occipital à ressonância magnética (T2) ao nível dos núcleos da base

utilizadas seqüências espectroscópicas com tempo de eco (TE) curto, ou seja, 20mseg. O tempo de repetição (TR) escolhido foi de $1500 \mathrm{mseg}$, associado à supressão do sinal dominante da água ${ }^{18}$. Para seqüência de pulso, optou-se pela técnica PRESS ("point-resolved spectroscopy"). A análise da curva espectral foi feita através de um software específico, mediante o qual se consegue localizar os picos de cada metabólito conforme a freqüência espectral correspondente $2,8,10,11,12,16,27,29,30$.

Em virtude da intensa sobreposição de Glx com o pico dos radicais metileno (amino-butirato, NAA e aspartato), uma integração manual foi processada entre 2,15 e 2,45ppm para o pico de Glx, com o objetivo de representar fielmente o nível de glutamina ${ }^{29}$. Este foi o único metabólito em que se avaliou a área envolvida abaixo da curva e não o pico, tendo em vista não formar um pico único como os outros metabólitos analisados (Figura 2).

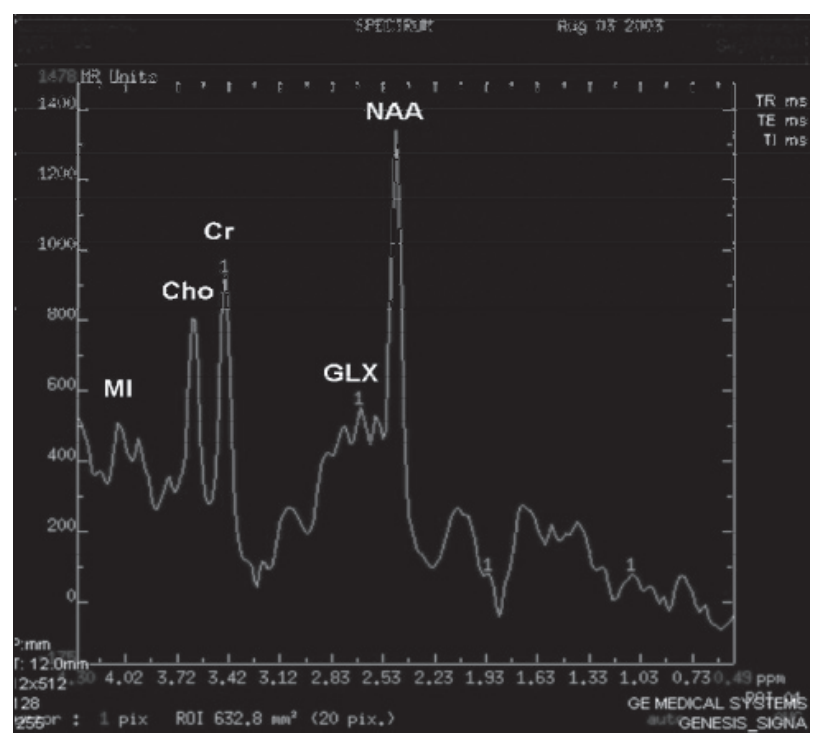

FIGURA 2 - Curva espectroscópica de indivíduo do grupo controle 
Após a determinação de cada pico a ser estudado e seus respectivos valores em unidades de ressonância (UR), foram calculados os índices absolutos para MI, Cho e Glx em relação a $\mathrm{Cr}$, usada como padrão de comparação. $\mathrm{O}$ grupo controle foi usado como referência da normalidade em função da técnica de ressonância magnética utilizada neste estudo.

O diagnóstico espectroscópico da encefalopatia hepática foi estabelecido segundo os critérios de $\operatorname{Ross}^{27}$, ou seja, quando os pacientes apresentavam o índice $\mathrm{MI} / \mathrm{Cr}$ e $\mathrm{Cho} / \mathrm{Cr}$ dois desvios-padrão abaixo dos controles $(\mathrm{MI} / \mathrm{Cr}$ e $\mathrm{Cho} / \mathrm{Cr}<$ média -2 desvios-padrão do grupo controle) com ou sem o índice Glx/Cr um desvio-padrão acima dos controles $(\mathrm{Glx} / \mathrm{Cr}>$ média +1 desvio-padrão do grupo controle). Esses critérios foram comparados com o teste padrão (testes neuropsicométricos) para avaliação dos índices de qualidade da espectroscopia no diagnóstico da encefalopatia hepática.

Para fins de comparação, os indivíduos avaliados foram divididos em três grupos distintos, de acordo com exame clínico e testes neuropsicométricos (Tabela 1) em: Grupo 1 (GR1): pacientes com EH ( $\mathrm{n}=13$ ); Grupo 2 (GR2): pacientes sem EH ( $n=12)$; Grupo 3 (GRC): grupo controle - voluntários sadios $(\mathrm{n}=30)$

TABELA 1 - Idade e sexo dos grupos estudados

\begin{tabular}{lcccc}
\hline Idade & \multicolumn{3}{c}{} \\
\hline Grupo & $\mathbf{n}$ & Min-máx & Mediana & Média \pm DP \\
\hline GR1 & 13 & $31,00-70,00$ & 50,00 & $51,15 \pm 10,27$ \\
GR2 & 12 & $17,00-60,00$ & 48,00 & $46,17 \pm 11,03$ \\
GRC & 30 & $14,00-61,00$ & 33,50 & $36,43 \pm 12,41$ \\
\hline Sexo & Masculino & Feminino \\
\hline GR1 & $10(73,92 \%)$ & $3(23,08 \%)$ \\
GR2 & $9(75,00 \%)$ & $3(25,00 \%)$ \\
GRC & $16(53,33 \%)$ & $14(46,67 \%)$ \\
\hline
\end{tabular}

* GR1 = pacientes com encefalopatia hepática; GR2 = pacientes sem encefalopatia hepática; GRC = grupo controle

Os índices absolutos dos metabólitos (NAA/Cr, $\mathrm{MI} / \mathrm{Cr}$, $\mathrm{Cho} / \mathrm{Cr}$ e $\mathrm{Glx} / \mathrm{Cr}$ ) tanto no grupo controle como no grupo de estudo foram analisados conforme a distribuição dos valores na curva de Gauss e, então, foram testadas as diferenças significativas entre os grupos por meio do teste não-paramétrico de Kruskal-Wallis. O nível máximo de significância adotado foi de $5 \%(P<0,05)$. Quando houve significância estatística, os valores aparecem em negrito.

\section{RESULTADOS}

Dos 25 pacientes avaliados, 13 apresentavam encefalopatia hepática, sendo clinicamente aparente em apenas 1 paciente $(4 \%)$ por apresentar confusão mental, bradipsiquismo, alterações do sono e asterixis (EH grau II). Este paciente apresentou todos os testes neuropsicométricos alterados, inclusive o mini-exame do estado mental. A encefalopatia subclínica foi diagnosticada, portanto, em 12 pacientes (48\%).

Os outros 12 pacientes (48\%) avaliados neste estudo não apresentavam evidências de encefalopatia, bem como nenhum dos indivíduos do grupo controle, que foram submetidos à mesma seqüência de exames.

No GR1 a média de idade foi de 51,15 $\pm 10,27$ anos com mediana de 50 anos, sendo 10 homens $(73,92 \%)$ e 3 mulheres $(23,08 \%)$. No GR2 a média de idade foi de $46,17 \pm 11,03$ anos com mediana de 48 anos, sendo 9 homens ( $75 \%)$ e três mulheres (25\%). E no GRC a média de idade foi de $36,43 \pm 12,41$ anos com mediana de 33,5 anos, sendo 16 homens $(53,33 \%)$ e 14 mulheres $(46,67 \%)$. Os indivíduos do GRC eram mais jovens $(P<0,0001)$, não havendo diferença significativa na distribuição por sexo entre os grupos. Todos os indivíduos do GRC apresentaram testes neuropsicométricos normais. No GR1, oito pacientes $(61,53 \%)$ tinham $50 \%$ dos testes alterados; em quatro, $75 \%$ dos testes estavam anormais e em um todos os testes neuropsicométricos estavam alterados. No GR2, nove pacientes $(75 \%)$ apresentavam um teste $(25 \%)$ alterado e três apresentavam todos os testes neuropsicométricos normais, não se configurando o diagnóstico de encefalopatia (Tabela 2).

TABELA 2 - Diagnóstico de encefalopatia hepática clínica e subclínica no período pré-transplante hepático

\begin{tabular}{|c|c|c|c|c|}
\hline Pacientes & $\begin{array}{l}\text { Exame } \\
\text { clínico }\end{array}$ & MEEM & TNPS & Classificação EH \\
\hline 1 & Normal & Normal & $75 \%$ alterados & Subclínica \\
\hline 2 & Normal & Normal & $75 \%$ alterados & Subclínica \\
\hline 3 & Normal & Normal & $25 \%$ alterados & Ausente \\
\hline 4 & Normal & Normal & $25 \%$ alterados & Ausente \\
\hline 5 & Normal & Normal & $0 \%$ alterados & Ausente \\
\hline 6 & Normal & Normal & $25 \%$ alterados & Ausente \\
\hline 7 & Normal & Normal & $50 \%$ alterados & Subclínica \\
\hline 8 & Normal & Normal & $0 \%$ alterado & Ausente \\
\hline 9 & Normal & Normal & $75 \%$ alterados & Subclínica \\
\hline 10 & Normal & Normal & $25 \%$ alterados & Ausente \\
\hline 11 & Normal & Normal & $50 \%$ alterados & Subclínica \\
\hline 12 & Normal & Normal & $50 \%$ alterados & Subclínica \\
\hline 13 & Normal & Normal & $75 \%$ alterados & Subclínica \\
\hline 14 & Normal & Normal & $50 \%$ alterados & Subclínica \\
\hline 15 & Normal & Normal & $50 \%$ alterados & Subclínica \\
\hline 16 & Normal & Normal & $50 \%$ alterados & Subclínica \\
\hline 17 & Normal & Normal & $50 \%$ alterados & Subclínica \\
\hline 18 & Normal & Normal & $25 \%$ alterados & Ausente \\
\hline 19 & Normal & Normal & $25 \%$ alterados & Ausente \\
\hline 20 & Normal & Normal & $50 \%$ alterados & Subclínica \\
\hline 21 & Normal & Normal & $25 \%$ alterados & Ausente \\
\hline 22 & Normal & Normal & $25 \%$ alterados & Ausente \\
\hline 23 & Alterado & Alterado & $100 \%$ alterados & Clínica \\
\hline 24 & Normal & Normal & $0 \%$ alterado & Ausente \\
\hline 25 & Normal & Normal & $25 \%$ alterados & Ausente \\
\hline
\end{tabular}

NOTA: O diagnóstico da encefalopatia hepática é considerado na presença de pelo menos $50 \%$ dos testes neuropsicométricos alterados

O mini exame do estado mental (MEEM) foi realizado em todos os pacientes e encontrava-se alterado em um paciente do GR1. O teste de trilhas A e B estava alterado em 17 pacientes (68\%), sendo 12 do GR1 e 5 do GR2. O subteste de dígitos do WAIS-R encontrava-se anormal em quatro pacientes (16\%), sendo todos do GR1. O teste de fluência verbal (FAR) teve resultado anormal em 15 pacientes (60\%), sendo 11 do GR1 e 4 do GR2. O teste do desenho do relógio estava anormal em 5 pacientes (20\%), sendo todos do GR1 (Tabela 3). 
TABELA 3 - Resultado do mini-exame do estado mental (MEEM) e dos testes neuropsicométricos (TNPS) no período pré-transplante hepático

\begin{tabular}{|c|c|c|c|c|c|}
\hline Paciente & МЕEM & FAR & Dígitos & Trilhas & Relógio \\
\hline 1 & - & + & - & + & + \\
\hline 2 & - & + & + & + & - \\
\hline 3 & - & - & - & + & - \\
\hline 4 & - & - & - & + & - \\
\hline 5 & - & - & - & - & - \\
\hline 6 & - & + & - & - & - \\
\hline 7 & - & + & + & - & - \\
\hline 8 & - & - & - & - & - \\
\hline 9 & - & + & - & + & + \\
\hline 10 & - & - & - & + & - \\
\hline 11 & - & - & - & + & + \\
\hline 12 & - & + & - & + & - \\
\hline 13 & - & + & - & + & + \\
\hline 14 & - & + & - & + & - \\
\hline 15 & - & + & - & + & - \\
\hline 16 & - & + & - & + & - \\
\hline 17 & - & - & - & + & - \\
\hline 18 & - & - & - & - & - \\
\hline 19 & - & - & - & - & - \\
\hline 20 & - & - & + & + & - \\
\hline 21 & - & + & - & - & - \\
\hline 22 & - & - & - & + & - \\
\hline 23 & + & + & + & + & + \\
\hline 24 & - & - & - & - & - \\
\hline 25 & - & - & - & + & - \\
\hline
\end{tabular}

NOTA: (-) teste normal, $(+)$ teste alterado

As dosagens de sódio e potássio plasmáticos, no pré-operatório, foram normais em todos os pacientes e controles

A avaliação espectroscópica dos metabólitos cerebrais (MI, Cho e Glx) entre pacientes e controles apresentou diferenças significativas. O índice médio de NAA/Cr nos pacientes do GR1 foi $1,92 \pm 0,24$, não apresentando diferença estatística em relação aos pacientes do GR2 e GRC, cujos índices foram $1,91 \pm 0,22$ e $1,89 \pm 0,28$, respectivamente (teste de Kruskal-Wallis, $P=0,9126$ ). Em relação ao $\mathrm{MI} / \mathrm{Cr}$, o índice médio nos pacientes do GR1 foi $0,49 \pm 0,10$ e nos pacientes do GR2 foi $0,56 \pm 0,17$, sendo em ambos os grupos inferior a dois desvios-padrão do índice médio do GRC, 0,83 $\pm 0,13$ (teste de Kruskal-Wallis, $P<0,0001$ ). Resultados similares foram observados para o índice médio de Cho/Cr: 0,54 $\pm 0,15$ nos pacientes do GR $1 ; 0,67 \pm 0,13$ nos pacientes do GR2 e 0,77 $\pm 0,09$ no GRC (teste de KruskalWallis, $P=0,0001$ ). A diferença no índice médio do $\mathrm{Glx} / \mathrm{Cr}$ entre os grupos foi menor, porém igualmente significativa: $1,12 \pm 0,26$ no GR $1 ; 1,00 \pm 0,28$ no GR 2 e $0,89 \pm 0,19$ no GRC (teste de Kruskal-Wallis, $P=0,0146$ ).

Em análise geral, o índice $\mathrm{MI} / \mathrm{Cr}$ encontrava-se depletado em $64 \%$ dos pacientes, o $\mathrm{Cho} / \mathrm{Cr}$ em $48 \%$, enquanto a $\mathrm{Glx} / \mathrm{Cr}$ encontrava-se acima dos controles em 52\% dos pacientes (Gráfico 1).

Comparando-se os grupos dois a dois, pôde-se observar diferenças significativas (Tabela 4).

Para validar os critérios quantitativos de Ross no diagnóstico espectroscópico da encefalopatia hepática, foram calculados os índices de qualidade da espectroscopia, usando como "padrão-ouro" os testes neuropsicométricos. Dos 13 pacientes com encefalopatia pelos testes neuropsi-

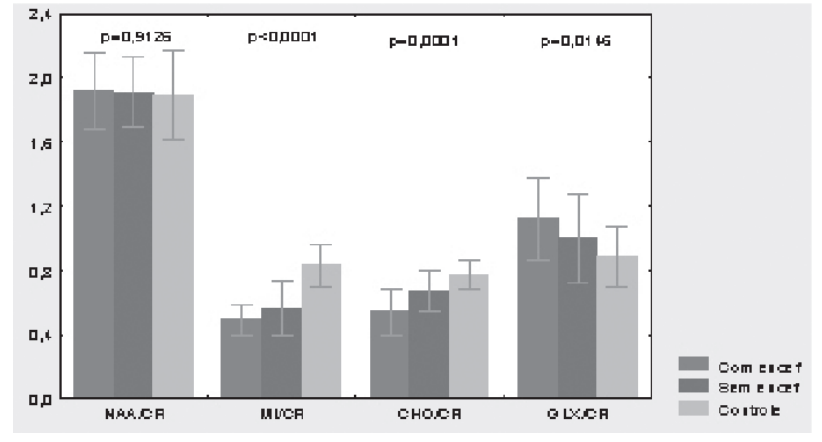

* Teste não-paramétrico de Kruskal-Wallis

GRÁFICO 1 - Médias dos metabólitos cerebrais avaliados pela espectroscopia

TABELA 4 - Comparação dos grupos 2 a 2 no pré-operatório

\begin{tabular}{cccc}
\hline \multirow{2}{*}{ Variável } & \multicolumn{3}{c}{ Comparações dos grupos 2 a 2 - valor de $\boldsymbol{P}$} \\
\cline { 2 - 4 } & GR1 x GR2 & GR1 x GRC & GR2 x GRC \\
\hline $\mathrm{MI} / \mathrm{CR}$ & 0,2310 & $<\mathbf{0 , 0 0 0 1}$ & $\mathbf{0 , 0 0 0 1}$ \\
$\mathrm{CHO} / \mathrm{CR}$ & $\mathbf{0 , 0 3 1 5}$ & $\mathbf{0 , 0 0 0 1}$ & $\mathbf{0 , 0 2 5 8}$ \\
$\mathrm{GLX} / \mathrm{CR}$ & 0,2207 & $\mathbf{0 , 0 0 4 8}$ & 0,1595 \\
\hline
\end{tabular}

(*) Teste não-paramétrico de Kruskal-Wallis

Os valores de $P$ em negrito indicam a rejeição da hipótese nula no nível de significância de 5\%. Para estes casos, pode-se afirmar que existe diferença significativa entre os grupos comparados

cométricos, a espectroscopia foi positiva em $8(61,5 \%)$ e negativa em 11 dos 12 pacientes sem encefalopatia $(91,6 \%)$. Um paciente apresentava critérios espectroscópicos para diagnóstico de encefalopatia, apesar de não apresentar alterações nos testes neuropsicométricos. Cinco pacientes apresentavam espectroscopia falso-negativa, acarretando valor preditivo negativo (VPN) baixo. Quando se utilizaram os critérios de Ross para diagnóstico espectroscópico da encefalopatia, a sensibilidade (S) foi de $61,54 \%$, a especificidade (E) de 91,67\%, o valor preditivo positivo (VPP) de $88,89 \%$, o VPN de $68,75 \%$ e a precisão de $76 \%$.

Analisando-se isoladamente cada fração (MI/Cr, Cho/ $\mathrm{Cr}$ e $\mathrm{Glx} / \mathrm{Cr}$ ) para diagnóstico da encefalopatia, pôde-e perceber algumas mudanças em relação aos índices de qualidade da espectroscopia. No caso da espectroscopia positiva apenas para $\mathrm{MI} / \mathrm{Cr}(<$ média - dois desvios-padrão do grupo controle), verificou-se aumento da sensibilidade (76,92\%), com redução da especificidade (50\%), do VPP $(62,5 \%)$, do VPN $(66,67 \%)$ e da precisão (64\%).

Quando se analisou apenas a $\mathrm{Cho} / \mathrm{Cr}$ como critério diagnóstico da encefalopatia na espectroscopia, observaramse resultados mais parecidos aos obtidos com a utilização dos critérios de Ross, porém com maior sensibilidade e menor especificidade. Nesse caso, a sensibilidade foi de $76,92 \%$, especificidade de $83,33 \%$, VPP de $83,33 \%$, VPN de $76,92 \%$ e precisão de $80 \%$.

A avaliação isolada do $\mathrm{Glx} / \mathrm{Cr}$ como critério diagnóstico da encefalopatia mostrou índices de qualidade inferiores às demais avaliações, com sensibilidade de $61,54 \%$, especificidade de $58,33 \%$, VPP de $61,54 \%$, VPN de $58,33 \%$ e acurácia de $60 \%$. 


\section{DISCUSSÃO}

A encefalopatia hepática intriga a medicina desde os tempos de Hipócrates, permanecendo até os dias de hoje sem compreensão dos mecanismos fisiopatológicos precisos que determinam seu aparecimento. Igualmente intrigante, são os efeitos que a encefalopatia hepática pode ocasionar no cérebro dos pacientes com hepatopatia crônica ao longo dos anos.

O caráter metabólico "reversível" da síndrome foi largamente difundido, sendo corroborado pela evidente melhora clínica percebida em pacientes com encefalopatia submetidos aos mais variados tratamentos médicos propostos na literatura. Porém, nos últimos anos, esta reversibilidade tem sido posta em xeque por autores como KRIEGER et al. ${ }^{12}$, que demonstraram a presença de déficits neurológicos persistentes a despeito do tratamento clínico da encefalopatia hepática em cirróticos, sugerindo distúrbio neurodegenerativo lentamente progressivo. Alguns relatos posteriores confirmaram estes déficits neurológicos, relacionando-os à deposição de manganês nos núcleos da base em pacientes com derivações portossistêmi$\operatorname{cas}^{21}$. Como se não bastasse, existem evidências crescentes de que a atrofia fronto-parietal é muito prevalente nos cirróticos, independente dos efeitos do álcool.

Sabe-se que a encefalopatia hepática subclínica pode afetar $44 \%$ a $70 \%$ dos cirróticos, dependendo dos testes e da população estudada. Isto representaria grande número de pacientes que, teoricamente, poderiam apresentar déficits neurológicos permanentes se não diagnosticados precocemente. No presente estudo, a prevalência da encefalopatia subclínica foi de $48 \%$.

A história natural da encefalopatia hepática subclínica não é bem conhecida. O tempo de progressão e aparecimento de alterações clínicas identificáveis é muito discutido na literatura, bem como se há impacto benéfico ou não do tratamento clínico precoce no sentido de se evitar a deterioração das funções cerebrais ao longo do tempo. O diagnóstico da encefalopatia subclínica tem como "padrão-ouro" a realização de testes neuropsicométricos. O problema na sua utilização isolada é que os resultados não são específicos desse distúrbio neurológico; podem ser confundidos com diversos outros fatores que interferem na função neurológica dos hepatopatas, como quadros demenciais, além de sofrerem interferências da idade e escolaridade dos pacientes. Os testes neuropsicométricos utilizados neste estudo foram escolhidos pela facilidade de aplicação e por serem breves e sensíveis para detectar alterações de atenção, raciocínio, funções espaciais e memória, localizadas predominantemente nos lobos frontal e parietal, sabidamente os mais acometidos na encefalopatia hepática.

Com a introdução da ressonância magnética nos estudos da encefalopatia, evidenciaram-se alterações na hidratação e na osmolaridade cerebral, permitindo avanços no entendimento neuroquímico da síndrome. A espectroscopia cerebral por ressonância magnética permite o estudo in vivo e não-invasivo dos processos metabólicos cerebrais envolvidos, podendo ser usada com alta especificidade no diagnóstico da encefalopatia, em conjunto com os testes neuropsicométricos que têm alta sensibilidade. A espectroscopia é capaz de detectar alterações da bioquímica cerebral, incluindo medida dos metabólicos cerebrais osmoticamente ativos envolvidos no processo intrínseco de homeostase celular, incluindo acúmulo intracelular de água e conseqüente aparecimento da encefalopatia, como o mioinositol (MI), colina (Cho) e glutamina-glutamato (Glx).

A concentração destes metabólicos intracelulares osmoticamente ativos altera-se com a hiperamonemia. O entendimento deste novo processo neuroquímico envolvendo a hidratação cerebral, bem como sua quantificação através da espectroscopia é que permite o diagnóstico preciso da encefalopatia, mesmo em estágios muito precoces. No presente estudo a especificidade da espectroscopia cerebral no diagnóstico da encefalopatia foi de $91,67 \%$.

Anormalidades metabólicas têm sido identificadas em diversas doenças neurológicas, como a doença de Alzheimer, na qual o marcador neuronal $\mathrm{N}$-acetilaspartato está depletado. Em contraste, a encefalopatia hepática é caracterizada por decréscimo substancial no mio-inositol e colina, associado a aumento tardio de glutamina-glutamato. Esta tríade característica de alterações não é detectada em nenhuma outra encefalopatia.

A despeito dos achados espectroscópicos positivos, o papel do MI e da Cho no cérebro humano e especialmente em encefalopatia hepática, não está completamente esclarecido. Na disfunção crônica do fígado, a diminuição da atividade do ciclo da uréia parece ser a responsável pela elevação da glutamina cerebral, cuja síntese é aumentada nos astrócitos. O MI, localizado exclusivamente nos astrócitos, tem papel fundamental na regulação do volume destas células, sendo considerado marcador astrocitário. Assim sendo, o edema das células da glia induzido pela amônia (não metabolizada no fígado doente) induz alta concentração celular de glutamina e liberação compensatória de MI desencadeando a encefalopatia. Neste mesmo raciocínio pode-se encaixar a Cho, que é um metabólito osmoticamente ativo presente na mielina, membranas celulares e outros complexos cerebrais lipídicos. É provável que, neste estudo, a depleção de Cho não tenha sido tão acentuada quanto à do MI em virtude de se ter utilizado área cerebral que envolve substância branca e cinzenta, e a Cho é exclusivamente encontrada na substância branca cerebral.

Neste contexto, algumas perguntas surgem ainda sem respostas definitivas: a encefalopatia realmente pode causar alterações cerebrais permanentes e em que momento isto começa a ocorrer? São realmente importantes o diagnóstico e a terapêutica precoces no curso da doença? A encefalopatia subclínica, freqüentemente negligenciada na prática clínica diária, também pode causar déficits neurológicos permanentes? O tratamento clínico reverte eficazmente estes efeitos ou apenas o transplante hepático seria a solução definitiva?

Todas estas perguntas ainda não podem ser completamente respondidas, carecendo-se de mais pesquisas para um parecer definitivo sobre o assunto.

\section{CONCLUSÃO}

As alterações metabólicas cerebrais que ocorrem precocemente no curso da encefalopatia hepática podem ser demonstradas pela espectroscopia, permitindo diagnóstico preciso antes mesmo do aparecimento de sinais e sintomas clínicos 
Schulz GJ, Coelho JCU, Matias JEF, Campos ACL, Schulz DD, Bertoldi GA. Detection of subclinical hepatic encephalopathy by magnetic resonance spectroscopy. ABCD Arq Bras Cir Dig 2007; 20(2):81-6.

ABSTRACT - Background - Hepatic encephalopathy is a common neuro-psychiatric abnormality in liver cirrhosis associated with typical changes of cerebral metabolite pattern, such as a decrease of myo-inositol and cholina and increase of glutamine-glutamate, observed by magnetic resonance spectroscopy. Aim - To determine cerebral metabolite ratios in liver cirrhosis patients with early stages of hepatic encephalopathy. Methods - Twenty-five patients with chronic hepatic failure from Liver Transplantation Unit of the Federal University of Paraná were studied with clinical evaluation and magnetic resonance spectroscopy. Localized magnetic resonance spectra were acquired in the occipital gray/white matter regions. Thirty healthy volunteers were also subjected to the same evaluations, making up the control group. Results - Subclinical hepatic encephalopathy was diagnosed in 12 patients $(48 \%)$. MI/Cr ratios were significantly reduced in cirrhotic patients with hepatic encephalopathy when compared to the control group $(0,49 \pm 0,10$ vs. $0,83 \pm 0,13 ; P<0,0001)$. In addition, patients with encephalopathy, showed reduction of the $\mathrm{Cho} / \mathrm{Cr}$ ratio $(0,54 \pm 0,15$ vs. $0,77 \pm 0,09 ; P=0,0001)$ and an elevated $\mathrm{Glx} / \mathrm{Cr}$ ratio $(1,12 \pm 0,26$ vs. $0,89 \pm 0,19 ; P=0,0146)$. The Ross's quantitive criterions for spectroscopic diagnoses of hepatic encephalopathy (MI/Cr and Cho/Cr below than $2 \mathrm{SD}$ of controls) demonstrated a sensibility of $61,54 \%$, specificity of $91,67 \%$ and accuracy of $76 \%$. Cho/Cr ratio was considered the best parameter. Conclusion - Cerebral metabolic alterations that occur at an early stage of hepatic encephalopathy may be demonstrated through magnetic resonance spectroscopy, allowing a precise diagnosis before the appearance of clinical signs and symptoms.

HEADINGS - Hepatic encephalopathy. Brain diseases. Magnetic resonance spectroscopy. Magnetic resonance.

\section{REFERÊNCIAS}

1. Adams RD, Victor M, Ropper AH. Special techniques for neurologic diagnosis. In: Adams RD, Victor M, Ropper AH, editors. Principles of neurology. 6th ed. New York: McGraw-Hill; 1997. p.33-8.

2. Bárány M, Langer BG, Glick RP, Venkatasubramanian PN, Wilbur AC, Spigos DG. In vivo H-1 spectroscopy in humans at 1,5T. Radiology. 1988;167:83944.

3. Bertolucci RG, Brucki SM, Campacci SR, Juliano Y. O mini-exame do estado mental em uma população geral. Arq Neuropsiquiatr. 1994;52;1-7.

4. Brucki SM, Malheiros SM, Okamoto IH, Bertolucci PH. Dados normativos para o teste de fluência verbal categoria animais em nosso meio. Arq Neuropsiquiatr. 1997;55:56-61.

5. Folstein MF, Folstein SE, McHugh PR. Mini-mental state - a practical method for grading the cognitive state of patients for the clinician. J Psychiat Res. 1975;12:189-98.

6. Geissler A, Lock G, Frund R, Held P, Hollerbach S, Andus T, et al. Cerebral abnormalities in patients with cirrhosis detected by proton magnetic resonance spectroscopy and magnetic resonance imaging. Hepatology. 1997;25:48-54.

7. Gitlin N. Subclinical portal-systemic encephalopathy. Am J Gastroenterol. 1998;83:8-11.

8. Häussinger D, Laubenberger J, Dahl S, Ernst T, Bayer S, Langer M, et al. Proton magnetic resonance sepectrocopy on human brain myo-inositol in hypo-osmalarity and hepatic encephalopathy. Gastroenterology. 1994;107:1475-80.

9. Hockerstedt K, Kajaste S, Isoniemi H, Muuronen A, Raininko R, Seppalainen AM, et al. Tests of encephalopathy before and after liver transplantation. Transplant Proc. 1992;22:1576-8.

10. Kreis R, Farrow N, Ross BD. Diagnosis of hepatic encephalopathy by proton magnetic resonance spectroscopy. Lancet. 1990;336:635-6.

11. Kreis R, Ross BD, Farrow NA, Ackerman Z. Metabolic disorders of the brain in chronic hepatic encephalopathy detected with H-1 MR spectroscopy. Radiology. 1992;182:19-27.

12. Krieger S, Jauss M, Jansen O, Theilmann L, Geissler M, Krieger D. MRI findings in chronic hepatic encephalopathy depend on portossystemic shunt: results of a controlled prospective clinical investigation. J Hepatol. 1997;27:121126.

13. Krieger D, Krieger S. Markers of reversible hepatic encephalopathy. Neurology. 1997;49:1187-8.

14. Laubenberger J, Häussinger D, Bayer S, Gufler H, Hennig J, Langer M. Proton magnetic resonance spectroscopy of the brain in symptomatic an asymptomatic patients with liver cirrhosis. Gastroenterology. 1997;112:1610-6.

15. Lezak MD. Neuropsychological assessment. 3rd ed. New York: Oxford; 1997.

16. Luyten PR, Den Hollander JA. Observation of metabolites in the human brain by MR spectroscopy. Radiology. 1986;161:795-8.
17. Moll J, Oliveira-Souza R, Moll FT, Bramati IE, Andreiuolo PA. The cerebral correlates of set-shifting. Arq Neuropsiquiatr. 2002;60:900-5.

18. Näegele T, Grodd W, Viebahn R, Seeger U, Klose U, Seitz D, et al. MR imaging and $1 \mathrm{H}$ spectroscopy of brain metabolics in hepatic encephalopathy: time-course of renormalization after liver transplantation. Radiology. 2000;216:683-91.

20. Neuberger J, McMaster P. Liver transplantation - indications. In: Blumgart LH, editor. Surgery of the liver and billiary tract. 1994;2:1801-10.

20. Oppong KNW, Al-Mardini H, Thick M, Record CO. Oral glutamine challenge in cirrhotic patients pre e post-liver transplantation: a psychometric and analyzed EEG study. Hepatology. 1997;26:870-6.

21. Pujol A, Pujol J, Graus F, Rimola A, Peri J, Mercader JM, et al. Hyperintense globus pallidus on T1-weigthed MRI in cirrhotic patients is associated with severity of liver failure. Neurology. 1993;43:65-9.

22. Quero JC, Hartmann IJ, Meulstee J, Hop WC, Schalm SW. The diagnosis of subclinical hepatic encephalopathy in patients with cirrhosis using neuropsychological tests and automated electroencephalogram analysis. Hepatology. 1996;24:556-60.

23. Rehnström S, Simert G, Hanssons JA, Johson G, Vang J. Chronic hepatic encephalopathy. A psychometrical study. Scand J Gastroenterol. 1977;12:30511.

24. Romero-Gómez M, Boza F, Garcia-Valdecasas MS, Garcia E, Aguilar-Reina J. Subclinical hepatic encephalopathy predicts the development of overt hepatic encephalopathy. Am J Gastroenterol. 2001;96:2718-23.

25. Rosa H. Encefalopatia hepática. In: Kalil AN, Coelho J, Strauss E, editores. Fígado e vias biliares: clínica e cirurgia. Rio de Janeiro: Revinter; 2001. p.102-9.

26. Ross BD, Kreis R, Ernst T. Clinical tools for the 90s: magnetic resonance spectroscopy and metabolite imaging. Eur J Radiol. 1993;14:128-40.

27. Ross BD, Jacobson S, Villamil F, Korula J, Kreis R, Ernst T, et al. Subclinical hepatic encephalopaty: proton MR spectroscopic abnormalities. Radiology. 1994;193:457-63.

28. Sleisenger M, Friedman L, Feldman M. Gastrointestinal bleeding. In: Sleisenger and Fordtran's gastrointestinal and liver disease. Philadelphia: WB Saunders; 1998.p.162-84.

29. Spahr L, Vingerhoets F, Lazeyras F, Delavelle J, Dupasquier R, Giostra E, et al. Magnetic resonance imaging and proton spectroscopic alterations correlate with parkinsonian signs in patients with cirrhosis. Gastroenterology. 2000;119:774-81.

30. Thomas MA, Huda A, Guze B, Curran J, Bugbee M, Fairbanks L, et al. Cerebral 1H MR spectroscopy and neuropsychologic status of patients with hepatic encephalopathy. Am J Roentgenol. 1998;171:1123-30.

31. Wechsler D, Wais-R. Manual. The psychological corporation Harcout Brave Jovanovich; 1981.

Conflito de interesse: não há

Fonte financiadora: não há

Recebido para publicação em: 22/01/2007

Aceito para publicação em: 15/04/2007 